\title{
Fluxo Reverso no Duto Venoso: Nova Perspectiva na Detecção de Anomalias Cromossômicas
}

\author{
Reverse Blood Flow in Ductus Venosus: New Perspective in Detection of \\ Chromosomal Abnormalities \\ Carlos Geraldo Viana Murta, Antônio Fernandes Moron, \\ Màrcio Augusto Pinto de Ávila, Luiz Cláudio França
}

\section{RESUM0}

Objetivo: estudar o duto venoso mediante o emprego do Doppler colorido e pulsátil com a finalidade de rastrear anomalias cromossômicas entre a $10^{a}$ e $14^{a}$ semanas de gestação. Métodos: a Dopplerfluxometria referente ao duto venoso e a medida da translucência nucal (TN) precederam a biopsia de vilo corial em 26 gestações. A suspeita de defeitos cromossomicos baseou-se nos seguintes critérios: fluxo ausente ou reverso durante a contração atrial no duto venoso e translucência nucal maior ou igual a $3 \mathrm{~mm}$. Verificaram-se a sensibilidade, a especificidade, o valor preditivo positivo e o negativo para cada um dos itens acima.

Resultados: ocorreram 9 casos de anomalias cromossômicas ( 3 trissomias do 21, 2 trissomias do 13, 1 trissomia do 9, 1 trissomia do 22, 1 triploidia e 1 monossomia do cromossomo X). $\mathrm{Na}$ totalidade dos casos o fluxo no duto venoso, durante a contração atrial, foi ausente (1 caso) ou reverso (8 casos), com sensibilidade de 100\%. No grupo de fetos normais (17 casos), a avaliação única apresentou alteração no Doppler (especificidade de 94\%). Concernente à medida da TN, a sensibilidade e a especificidade foram de $88 \%$ e 76\%, respectivamente. Conclusão: os resultados preliminares sugerem que a presença de anomalias cromossômicas pode ser fortemente suspeitada quando existir aumento da TN associado a fluxo ausente ou reverso no duto venoso durante a contração atrial. Especulamos que ambos os métodos sejam válidos no rastreamento dos defeitos cromossômicos.

PALAVRAS-CHAVE: Translucência nucal. Anomalias Cromossômicas. Dopplervelocimetria. Malformações fetais, Diagnóstico pré-natal.

\section{Introdução}

Uma série de pesquisas tem demonstrado significante aumento da translucência nucal (TN), entre a $10^{\text {a }}$ e $14^{\text {a }}$ semanas, associada a anormalidades cromossômicas. Os estudos assinalam que, se houver treinamento adequado, a utilização desse novo método diagnóstico

Disciplina de Obstetrícia do Departamento de Tocoginecologia da UNIFESP- Escola Paulista de Medicina.

Correspondência:

Carlos G. V. Murta

Av. César Hilal - 1181/401 - Praia do Suá

29052-231 - Vitória - ES

Tel. (27) 227-2543 - Fax: (27) 397-1649

e-mail: cgvmurta@zaz.com.br poderia rastrear aproximadamente $80 \%$ dos fetos portadores da síndrome de Down, com 5\% de resultados falso-positivos ${ }^{1,2,3}$. Dessa forma, os pesquisadores têm considerado a medida da TN associada à idade materna como sendo procedimento efetivo de rastreamento de aneuploidias, particularmente trissomias. Observou-se que o aumento da TN pode igualmente associar-se a alterações cardiovasculares, pulmonares, síndromes gênicas, infecções congênitas, doenças metabólicas, hematológicas e múltiplos outros defeitos anatômicos na ausência de anormalidades cromossômicas $^{1,2,3,4}$.

As bases dos mecanismos fisiopatológicos 
para explicar esse marcador ultra-sonográfico transitório (TN) ainda não estão estabelecidas. Alguns eventos que ocorrem nessa etapa da prenhez poderiam, eventualmente, esclarecer o acúmulo transitório de líquido na região da nuca do feto. Causa provável diz respeito às alterações da drenagem linfática fetal e/ou, particularmente, a distúrbio hemodinâmico do feto (disfunção

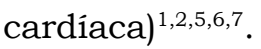

O feto possui 3 derivações anatômicas na sua circulação, com a finalidade de modificar a direção do sangue de modo a não ocorrer mistura entre o fluxo oxigenado, proveniente da placenta, e o de baixa saturação que retorna ao coração. O duto venoso é uma das 3 derivações exclusivas da circulação fetal que tem a função de conduzir diretamente o sangue bem oxigenado da veia umbilical para o coração ${ }^{8}$. Aproximadamente $50 \%$ do sangue oxigenado da veia umbilical penetra no sistema venoso hepatoportal e o restante dirige-se à veia cava inferior torácica através do duto venoso (primeira derivação). Dessa forma, a saturação de hemoglobina é a mesma no duto venoso e na veia umbilical ${ }^{9}$.

Mediante o emprego do Doppler colorido, o duto venoso revela-se como vaso pequeno e estreito que possui fluxo de alta velocidade, semelhante ao das artérias que atinge o forame oval sem misturarse com a corrente que insurge no átrio direito, pouco oxigenada ${ }^{10}$.

Estima-se que em 40 a 50\% das trissomias do cromossomo 21 e 90\% dos casos relativos à sindrome de Edwards ocorre algum tipo de cardiopatia. Nossa hipótese é que a onda velocimétrica no duto venoso poderá se alterar quando houver algum tipo de disfunção cardíaca e edema nucal, particularmente havendo defeito cardíaco, fato bastante comum entre os fetos com cromossomopatias ${ }^{11}$.

O objetivo deste trabalho foi avaliar a validade da Dopplervelocimetria do duto venoso na detecção de anomalias cromossômicas.

\section{Pacientes e Métodos}

O estudo teve caráter clínico e laboratorial. Foram analisados, por meio da ultra-sonografia, 120 fetos no primeiro trimestre de gestação (entre 10 e 14 semanas), de forma prospectiva (periodo de setembro de 1998 a março de 1999), nas cidades de São Paulo e Vitória (ES). Os exames foram realizados por dois ultra-sonografistas (C.G.V.M. e A.F.M.). A presente análise compreende 26 casos nos quais se realizou estudo citogenético (em 25 casos a análise do cariótipo foi realizada no material obtido por meio da biopsia de vilosidade coriônica e apenas 1 caso no produto de abortamento). Todos os fetos foram submetidos, além da ultra-sonografia de rotina, à medida da TN e à Dopplervelocimetria no duto venoso.

A medida da TN foi aferida mediante "corte" sagital do feto, o mesmo utilizado para a avaliação do CCN: no feto com a coluna posteriormente localizada, mediu-se o espaço hipoecogênico entre a pele e o tecido celular subcutâneo que recobre a coluna cervical.

O duto venoso foi identificado com Doppler colorido no figado fetal, aproximadamente entre o lobo direito e esquerdo, em um plano sagital e ventral, à direita do tronco do feto (Figura 1). A amostra de volume do Doppler pulsátil foi posicionada na região do istmo, imediatamente acima da veia umbilical (duto venoso proximal), local onde o fluxo torna-se mais brilhante e com tonalidade mais clara. A Figura 2 mostra que a onda de velocidade de fluxo do duto venoso é unidirecional e bifásica. O primeiro pico surge durante a sístole ventricular (S) e o segundo, na diástole ventricular (D). A incisura que aparece entre 2 ciclos representa o final da diástole e a contração atrial (a). Enfatizamos neste trabalho o comportamento da velocidade do fluxo, particularmente durante a fase "a" da onda velocimétrica. Considerando o pequeno comprimento do duto venoso, na análise da contração atrial, há de se tomar bastante cuidado para evitar contaminação com o fluxo da porção intra-hepática da veia umbilical, da veia hepática esquerda e da veia cava inferior.

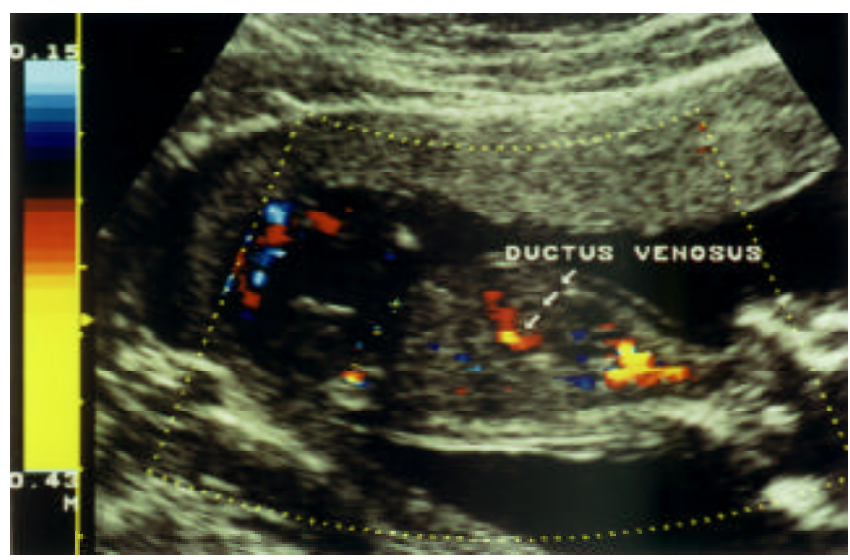

Figura 1 - Duto venoso visualizado num plano sagital de um feto com 12 semanas de gestação. Observa-se o aumento da velocidade do fluxo no duto venoso em relação à veia umbilical (tonalidade mais clara), decorrente do efeito "aliasing". 


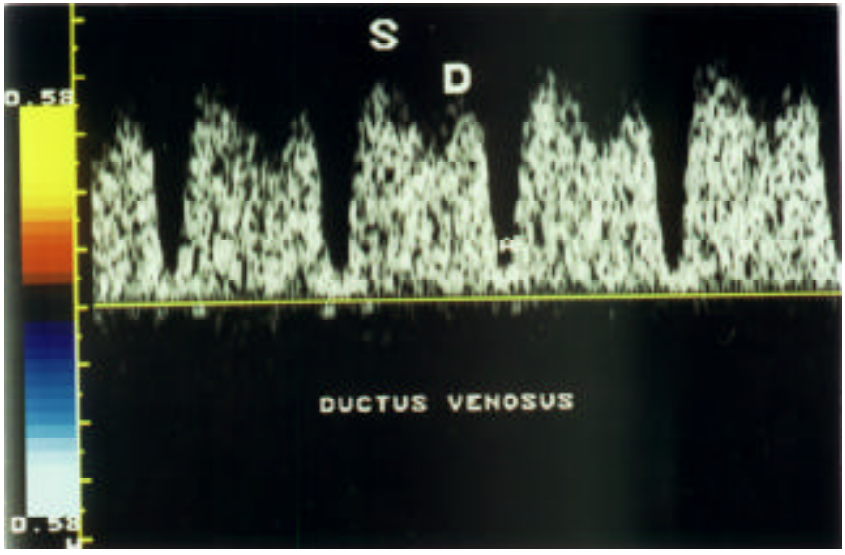

Figura 2 - Onda de velocidade de fluxo do duto venoso unidirecional e bifásica (feto de 12 semanas). 0 primeiro pico surge durante a sístole ventricular (S) e o segundo, na diástole ventricular (D). A incisura que aparece entre 2 ciclos representa o final da diástole e a contração atrial (a).

Em São Paulo o estudo foi realizado por via abdominal (aparelho Diassonic Synergi, com transdutor convexo de $3.5 \mathrm{MHz}$ ) e em Vitória na maioria das vezes por via vaginal (aparelho Toshiba $140 \mathrm{com}$ transdutor de $6.0 \mathrm{MHz}$ ). Os equipamentos têm capacidade de emitir simultaneamente, em mesmo tempo real, o ultra-som convencional (escala de cinza) e Doppler (colorido e pulsátil). O tamanho da amostra de volume foi ajustado em um nível mínimo para evitar interferência com os vasos adjacentes, mantendo-se entre 1 e $2 \mathrm{~mm}$. Para remover as interferências de baixas freqüências causadas pelo movimento das paredes dos vasos, utilizou-se filtro de 50 e $70 \mathrm{~Hz}$. Nos planos referidos acima, o ângulo entre o feixe ultrasônico e a direção do fluxo sempre foi menor que $30^{\circ}$. Foram obtidas 3 ondas velocimétricas com o máximo de clareza e amplitude (máxima velocidade possivel). O valor de cada parâmetro foi o resultado da média das 3 medidas.

Os resultados foram formulados em termos de suspeição ou não de anomalias cromossômicas. Para as aneuploidias foram adotados os seguintes critérios: para a medida da TN, ponto de corte maior ou igual a $3 \mathrm{~mm}$ e para o Doppler do duto venoso, velocidade "a" (durante contração atrial) ausente ou reversa. Calculamos a sensibilidade, a especificidade, o valor preditivo positivo e o valor preditivo negativo. $\mathrm{Na}$ análise estatística foi utilizado o teste exato de Fisher.

\section{Resultados}

Os resultados dos casos estudados estão representados na Tabela 1 . A média da idade materna foi 33 anos (variando de 17 a 43 anos). A média de idade no grupo portador de cromos- somopatias foi de 34 anos (variando de 17 a 43 anos), ao passo que no grupo que não apresentou alteração do cariótipo foi de 33 anos (variando de 24 a 41 anos). Com relação ao CCN, a média foi de $56,9 \mathrm{~mm}(38-82)$ e a idade gestacional média foi de 11 semanas e 5 dias. A distribuição do valor da TN nos 26 casos está exposta na Figura 3. Observamos 12 fetos que apresentaram TN igual ou maior que $3 \mathrm{~mm}$ e em 8 o cariótipo foi anormal, incluindo 3 trissomias do 21, 2 trissomias do 13, 1 trissomia do 9, 1 trissomia do 22, 1 triploidia e 1 monossomia do cromossomo X. Dos 14 fetos que exibiram TN menor que $3 \mathrm{~mm}$, apenas 1 (caso 15) apresentou anomalia cromossômica (trissomia do 22), resultando em uma sensibilidade de $87 \%$, especificidade de $76 \%$, valor preditivo positivo de $66 \%$ e valor preditivo negativo de $92 \%$. Ocorreram 4 casos de resultados falso-positivos, conforme pode-se verificar na Figura 3.

Tabela 1 - Resumo dos casos estudados

\begin{tabular}{|c|c|c|c|c|c|}
\hline Caso & $\begin{array}{l}\text { Idade } \\
\text { (anos) }\end{array}$ & $\begin{array}{c}\text { IG } \\
\text { (sem.) }\end{array}$ & $\begin{array}{c}\text { TN } \\
(\mathrm{mm})\end{array}$ & Veloc. "a" & Cariótipo \\
\hline 01 & 30 & 12 & 9.1 & Reversa & $47, X Y,+9$ \\
\hline 02 & 39 & 11 & 1.3 & Normal & $46, X X$ \\
\hline 03 & 36 & 12 & 4.1 & Reversa & $47, X X,+13$ \\
\hline 04 & 28 & 10 & 3.7 & Reversa & $47, X Y,+21$ \\
\hline 05 & 35 & 11 & 1.3 & Normal & $46, X X$ \\
\hline 06 & 33 & 11 & 2.0 & Normal & $46, X Y$ \\
\hline 07 & 43 & 11 & 4.2 & Reversa & $47, X X,+13$ \\
\hline 08 & 24 & 13 & 4.3 & Normal & $46, X X$ \\
\hline 09 & 38 & 14 & 6.2 & Reversa & $47, X X,+21$ \\
\hline 10 & 40 & 13 & 2.7 & Normal & $46, X Y$ \\
\hline 11 & 41 & 10 & 5.0 & Reversa & $69, X X X$ \\
\hline 12 & 27 & 10 & 3.6 & Normal & $46, X X$ \\
\hline 13 & 25 & 12 & 1.2 & Normal & $46, X Y$ \\
\hline 14 & 35 & 13 & 1.9 & Normal & $46, X Y$ \\
\hline 15 & 29 & 11 & 2.5 & Reversa & $47, X X,+22$ \\
\hline 16 & 43 & 10 & 3.1 & Ausente & $47, X X,+21$ \\
\hline 17 & 37 & 11 & 1.2 & Normal & $46, X X$ \\
\hline 18 & 37 & 10 & 1.0 & Normal & $46, X Y$ \\
\hline 19 & 38 & 12 & 1.7 & Normal & $46, X X$ \\
\hline 20 & 41 & 12 & 1.7 & Reversa & $46, X Y$ \\
\hline 21 & 33 & 11 & 1.0 & Normal & $46, X Y$ \\
\hline 22 & 27 & 14 & 2.6 & Normal & $46, X X$ \\
\hline 23 & 29 & 13 & 3.0 & Normal & $46, X Y$ \\
\hline 24 & 25 & 11 & 2.0 & Normal & $46, X Y$ \\
\hline 25 & 17 & 13 & 4.9 & Reversa & $45, X$ \\
\hline 26 & 35 & 13 & 5.0 & Normal & $46, X X$ \\
\hline
\end{tabular}

IG = Idade gestacional; TN = Translucência nucal;

Veloc. "a" = Velocidade do fluxo no duto venoso durante a contração atrial. 


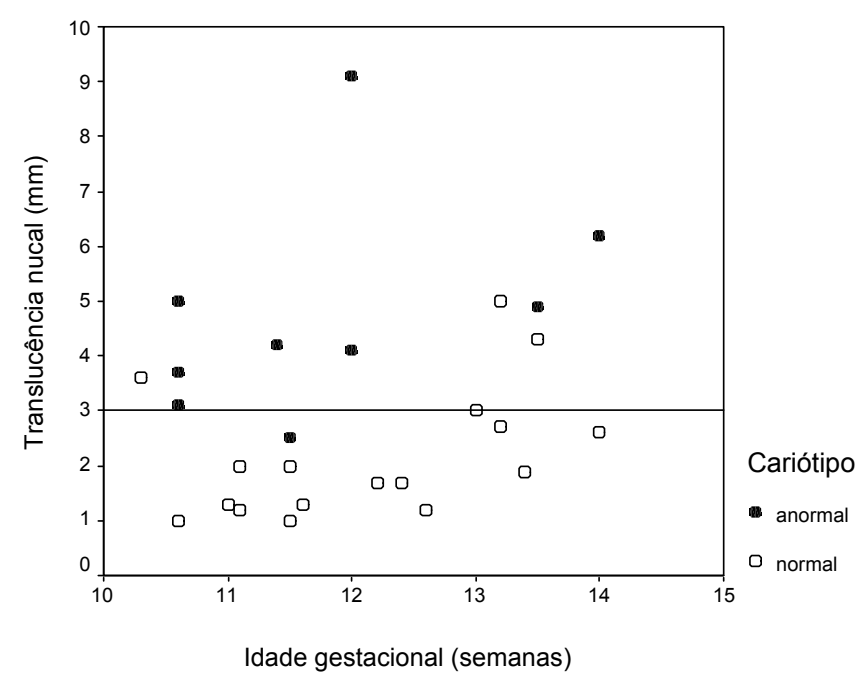

Figura 3 - Distribuição da medida da translucência nucal nos 26 casos estudados.

Em relação ao fluxo no duto venoso, em 16 dos 17 fetos com cariótipo normal, o fluxo foi normal durante a fase final do enchimento ventricular e contração atrial (apenas 1 caso de falso-positivo). Ao contrário, nos 8 casos com anomalias cromossômicas o fluxo foi reverso em 7 (Figura 4) e ausente em 1 caso (caso 16, Tabela 1). A onda de velocidade do fluxo do duto venoso foi obtida satisfatoriamente em todos os 26 casos, acrescentando em média 5 minutos ao tempo dispensado para obter um boa medida da TN.

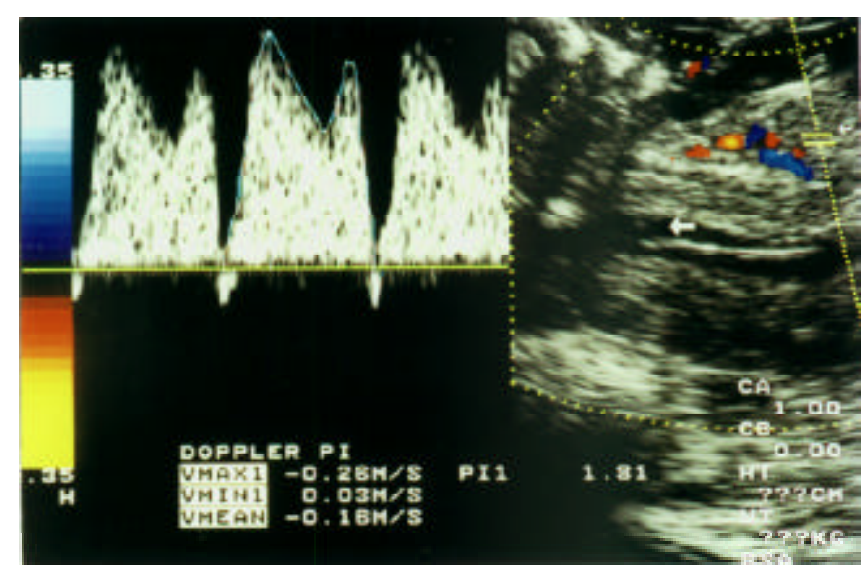

Figura 4 - Onda de velocidade do fluxo do duto venoso de aspecto trifásico devido à velocidade reversa durante a contração atrial em feto com 11 semanas de idade gestacional. 0 estudo citogenético concluiu por trissomia do cromossomo 22 (caso 15).

A sensibilidade do Doppler na detecção de anomalias cromossômicas foi de $100 \%$, com especificidade de $94 \%$. O valor preditivo positivo foi de $90 \%$ e o valor preditivo negativo de $100 \%$. A diferença entre os 2 grupos (cariótipo normal e anormal) foi significante, quando analisada estatisticamente pelo teste exato de Fisher $(p<0,0001)$.

\section{Discussão}

Os dados do presente estudo confirmam os resultados da literatura, os quais evidenciam que o aumento da TN aumenta o risco para anomalia cromossômica ${ }^{2,3}$, como também reforça a hipótese de que a disfunção cardíaca pode ser a base fisiopatológica do aumento da $\mathrm{TN}^{5}$.

A forma da onda de velocidade do fluxo no duto venoso é reflexo do gradiente de pressão entre o átrio direito e a veia umbilical. Sua alteração é sinal indireto de comprometimento do coração fetal. Recentes estudos demonstraram essa correlação tanto na segunda metade da gravide $z^{12,13}$, como no primeiro trimestre $\mathrm{e}^{5,14,15,16,17}$.

As alterações dos fluxos na veia umbilical, na veia cava inferior e no duto venoso revelam aumento de pressão ventricular durante a fase diastólica final e a contração atrial. A redução da velocidade do fluxo sangüíneo no duto venoso durante a contração atrial está associada a crescimento intra-uterino retardado ${ }^{13}$, malformação cardíaca ${ }^{12}$ e cromossomopatia ${ }^{5,14,15,16,17}$.

Montenegro et al. ${ }^{5}$ estudaram o Doppler do duto venoso e a medida da TN em 65 fetos entre 10 e 13 semanas de idade gestacional. Destes, 17 apresentaram TN acima de $3 \mathrm{~mm}$. Em relação ao duto venoso, todos os 5 casos portadores de cromossomopatias evidenciaram alteração na Dopplervelocimetria. O único parâmetro velocimétrico que se alterou foi a velocidade do fluxo durante a contração atrial, abaixo de $2 \mathrm{~cm} / \mathrm{s}$ $(\mathrm{p}<0.001)$. Os autores acreditam que tanto o aumento da TN quanto a alteração velocimétrica no duto venoso ocorrem por disfunção do coração, e que a alteração do Doppler concorra para diminuir a taxa de falso-positivo da medida da TN no rastreamento de cromossomopatias.

Matias et al. ${ }^{16}$ relataram alterações do retorno venoso em 3 fetos que exibiram aumento da TN e anomalias cromossômicas reveladas no estudo citogenético. No primeiro caso, a Dopplervelocimetria mostrou aumento do fluxo reverso na veia cava inferior e presença de fluxo reverso no duto venoso durante a contração atrial. O cariótipo revelou trissomia do cromossomo 13. Avaliação de outro feto com 12 semanas de idade gestacional mostrou TN de $5.3 \mathrm{~mm}$ e fluxo reverso no duto venoso durante a contração atrial. O estudo citogenético revelou tratar-se de trissomia do 18 . O terceiro caso, com 12 semanas de gestação e TN de $10 \mathrm{~mm}$, apresentou defeito completo de septo atrioventricular, aumento do fluxo reverso na veia cava inferior e ausência de fluxo no duto venoso durante a contração atrial. O cariótipo foi compativel com trissomia do cromossomo 21 . 
Em casuística mais alargada, Matias et al. ${ }^{17}$ rastrearam 486 gravidezes entre 10 e 14 semanas. Dessas, 63 apresentavam anomalias cromossômicas (38 casos de trissomia do 21, 12 trissomias do 18,7 do 13, 3 casos de sindrome de Turner e 3 casos de triploidia). Em 57 dos 63 casos de aneuploidia $(90,5 \%)$ foi evidenciado fluxo ausente ou reverso no duto venoso durante a contração atrial. Essa mesma alteração foi encontrada em 13 casos (3,1\%) dos 423 fetos cromossomicamente normais, mas em 7 destes 13 casos foram demonstrados defeitos cardiacos de grande porte entre 14 e 16 semanas de idade gestacional. Esses dados reforçam a hipótese de que essa alteração velocimétrica reflete uma disfunção cardíaca, mesmo que seja transitória, visto que em um dos casos de síndrome de Down descrito por Matias et al. ${ }^{17}$, a medida da TN e a alteração do fluxo no duto venoso na $13^{\mathrm{a}}$ semana normalizaram-se na $15^{\mathrm{a}}$ semana de gestação. Caso semelhante foi publicado por Huisman e Bilardo ${ }^{18}$, condizente com a trissomia do 18 , que revelou fluxo reverso no duto venoso e aumento da TN com 13 semanas em gravidez gemelar na qual o outro gêmeo não evidenciava nenhuma alteração. Na $20^{a}$ semana tanto a velocidade no duto quanto à $\mathrm{TN}$ reverteram a normalidade.

Apesar do pequeno número de casos, os nossos resultados foram semelhantes aos de Matias et al. ${ }^{17}$. A experiência aqui referida mostrou que todos os fetos com aneuploidias exibiram alteração do fluxo durante a contração atrial. A possibilidade de essa anormalidade no duto venoso vislumbrar uma falha cardiaca precoce foi confirmada no caso 1 (trissomia 9), no qual, por meio da ultrasonografia vaginal na $12^{\mathrm{a}}$ semana, suspeitou-se da presença de comunicação interventricular, o que foi confirmado pelo estudo anatomopatológico. Neste caso, além do fluxo reverso no duto venoso foi evidenciado aumento do percentual de fluxo reverso na veia cava inferior e fluxos reversos na artéria e veia umbilical. A derradeira verificação (fluxo reverso na veia umbilical) está sendo descrita na literatura pela primeira vez.

$\mathrm{Na}$ presente investigação destaca-se também o caso 15, gestante de 28 anos, em que o feto apresentou TN de 2,5 $\mathrm{mm}, \mathrm{CCN}$ de 52,9, risco corrigido com base no programa da "Fetal Medicine Foundation" de 1/298, ou seja, abaixo do "ponto de corte" (1/250) considerado de risco. O estudo citogenético concluiu por trissomia do 22. Desta forma, é possível que a alteração da velocidade do fluxo no duto venoso, notadamente durante a fase diastólica final e na contração atrial, contribua com a medida da TN no rastreamento de anomalias cromossômicas, não apenas no sentido de diminuir a taxa de falso-positivo, como sugere Montenegro et al. ${ }^{5}$. Este caso permite-nos especular a possibilidade de o Doppler do duto venoso aumentar a sensibilidade da detecção das aneuploídias, com o proveito de isenção de qualquer dano para o feto.

No trabalho de Montenegro et $a 1 .{ }^{5}$, o único parâmetro velocimétrico alterado na onda de velocidade de fluxo do duto venoso nos casos de cromossomopatia foi a velocidade minima na contração atrial. Em investigação recente, Borrel et al. ${ }^{15}$ avaliaram a velocimetria no duto venoso em 534 fetos entre 10 e 18 semanas de gestação. Dentre os parâmetros estudados, o aumento do indice de pulsatilidade (IP) teve maior importância na detecção da sindrome de Down. Uma proporção substancial de fetos portadores de trissomia do 21 , $73 \%$ (8/11), apresentou aumento do IP, ao passo que no trabalho de Matias et al. ${ }^{17}$, apesar de o IP ter sido significantemente mais elevado no grupo de fetos com aneuploidias, a análise de regressão multivariada demonstrou que apenas a velocidade na contração atrial contribuiu de forma independente na discriminação de fetos cromossomicamente normais e anormais. Na nossa experiência, todos os casos com anormalidades cromossômicas caracterizaram-se pelo fluxo ausente ou reverso, além de considerar-se que fluxos reversos durante a contração atrial aumentam o IP do duto venoso.

Depreende-se que a medida da $\mathrm{TN}$, já considerada excelente meio de rastreamento de cromossomopatias, vem de ser adotada como rotina em diversos centros. Apesar dos bons resultados no que tange ao Doppler do duto venoso na detecção das aneuploidias, ainda necessita estudos mais substanciais. Os recentes trabalhos não permitem propor essa nova técnica como método diagnóstico. Relatamos os resultados prévios que permitem especular a importância deste exame ultrasonográfico no rastreamento de anomalias cromossômicas. Além disso, a alteração da onda de velocidade do fluxo desse vaso no $1^{\circ}$ trimestre da gestação poderá ser um sinal de alerta para a investigação de defeitos cardíacos.

Para que haja justificativa para busca dessas alterações fetais, faz-se necessário um estudo multicêntrico com amplo protocolo de rastreamento. No entanto, os 3 únicos trabalhos sobre duto venoso na detecção de cromossomopatias existentes na literatura, assim como o nosso, consideram este novo método ultra-sonográfico, não-invasivo, como sensivel e específico na indicação de risco para as anomalias cromossômicas no primeiro trimestre da gestação. Assim é que a velocimetria no duto venoso talvez possa associar-se ao exame da medida da $\mathrm{TN}$, notadamente no sentido de diminuir a taxa de resultados falso-positivos. 


\section{SUMMARY}

Purpose: to evaluate the possible value of pulsed and color Doppler of ductus venosus blood flow in the screening for chromosomal abnormalities at 10-14 weeks of gestation.

Methods: the ductus venosus flow velocity waveforms and the nuchal translucency (NT) thickness were obtained immediately before the chorionic villus sample in 26 pregnancies. We employed the following criteria for the suspicion of chromosomal defects: reverse or absent flow during atrial contraction and NT greater or equal to $3 \mathrm{~mm}$. We calculated the sensitivity, the specificity, the negative and positive predictive value for each of the above items.

Results: there were 9 chromosomal abnormalities ( 3 cases of trisomy 21, 2 cases of trisomy 13, 1 case of trisomy 9, 1 case of trisomy 22, 1 triploidy and 1 monosomy X). Abnormal ductus venosus flow was observed in all cases (sensitivity of $100 \%$ ). In the normal fetuses (17 cases) only 1 had abnormal flow (specificity of 94\%). Concerning NT, the sensitivity and the specificity were $88 \%$ and $76 \%$, respectively.

Conclusion: our preliminary results suggest that the presence of chromosomal abnormalities may be strongly suspected when an increased NT thickness is associated with an absent or reverse flow in the ductus venosus. We speculated that both methods are valid in the screening of chromosomal defects.

KEY WORDS: Nuchal translucency. Chromosomal abnormalities. Collor Doppler. Ductus venosus.

\section{Referências}

1. Nicolaides KH, Azar G, Snijders RJM, Gosden CM. Fetal nuchal edema: associated malformations and chromosomal defects. Fetal Diagn Ther 1992; 7:123-31.

2. Nicolaides K, Brizot ML, Snijders RJ. Fetal nuchal translucency: ultrasound screening for fetal trisomy in the first trimester of pregnancy. $\mathrm{Br} \mathrm{J}$ Obstet Gynecol 1994; 101:782-6.

3. Snijders RJM, Noble P, Sebire N, Souka A, Nicolaides $\mathrm{KH}$. UK multicentre project on assessment of risk of trisomy 21 by maternal age and fetal nuchal translucency thickness at 10-14 weeks of gestation. Lancet 1998; 352:343-6.

4. Souka AP, Snijders RJM, Novakov A, Soares W, Nicolaides KH. Defects and syndromes in chromosomally normal fetuses with increased nuchal translucency thickness at 10-14 weeks of gestation. Ultrasound Obstet Gynecol 1998; 11:391-400
5. Montenegro N, Matias A, Areias JC, Castelo S, Barros H. Increased fetal nuchal translucency: possible involvement of early cardiac failure. Ultrasound Obstet Gynecol 1997; 10:265-8.

6. Pajkrt E, Bilardo CM, Vanlith JMM, Mol BWJ, Bleker OP. Nuchal translucency measurement in normal fetuses. Obstet Gynecol 1995; 86:994-7.

7. Murta CGV, Moron AF, Ávila MAP. Anomalias cromossômicas e suas circunstâncias: condição cardiaca fetal mediada pelo duto venoso. Femina 1998; 26:641-6.

8. Huisman TWA, Stewart PA, Wladimiroff JW. Ductus venosus blood flow velocity wave-forms in a human fetus - a Doppler study. Ultrasound Med Biol 1992; 18:33-7.

9. Hecher K, Campbell S. Characteristics of fetal venous blood flow under normal circumstances and during fetal disease. Ultrasound Obstet Gynecol 1996; 7:68-83.

10.Kiserud T, Eik-Nes SH, Blaas HGK, Hellevik LR. Ultrasonographic velocimetry of the fetal ductus venosus. Lancet 1991; 338:1412-4.

11. Hyett JA, Noble PL, Snijders RJM, Montenegro N, Nicolaides KH. Fetal heart rate in trisomy 21 and other chromosomal abnormalities at 10-14 weeks of gestation. Ultrasound Obstet Gynecol 1996; $7: 239-44$.

12.Kiserud T, Eik-Nes SH, Hellevik LR, Blaas HG. Ductus venosus blood velocity changes in fetal cardiac diseases. J Matern Fetal Invest 1993; 3:15-20.

13.Kiserud T, Eik-Nes SH, Blaas HG, Hellevik LR, Simensen B. Ductus venosus blood velocity and the umbilical circulation in the seriously growthretarded fetus. Ultrasound Obstet Gynecol 1994; 4:109-14.

14.Areias JC, Matias A, Montenegro N, Brandão O. Early antenatal diagnosis of cardiac defects using transvaginal Doppler ultrasound: new perspectives? Fetal Diagn Ther 1998; 13:111-4.

15.Borrel A, Antolin E, Costa D, Farre MT, Martinez JM, Fortuny A. Abnormal ductus venosus blood flow in trisomy 21 fetuses during early pregnancy. Am J Obst Gynecol 1998; 179:1612-7.

16. Matias A, Montenegro N, Areias JC, Brandão O. Anomalous fetal venous return associated with major chromosomopathies in the late first trimester of pregnancy. Ultrasound Obstet Gynecol 1998; 11:209-13.

17. Matias A, Gomes C, Flack N, Montenegro N, Nicolaides KH. Screening for chromosomal abnormalities at 10-14 weeks: the role of ductus venosus blood flow. Ultrasound Obstet Gynecol $1998 ; 12: 380-4$.

18. Huisman TWA, Bilardo CM. Transient increase in nuchal translucency thickness and reversed enddiastolic ductus venosus flow in a fetus with trisomy 18. Ultrasound Obstet Gynecol 1997; 10:397-9. 